\title{
Editorial
}

\section{Fractal-Type Dynamical Behaviors of Complex Systems}

\author{
Viorel-Puiu Paun $\mathbb{D D}^{1}$ Maricel Agop $\mathbb{D}^{1},{ }^{2}$ Guanrong Chen, ${ }^{3}$ and Cristian Focsa $\mathbb{D}^{4}$ \\ ${ }^{1}$ Physics Department, Faculty of Applied Sciences, Polytechnica University of Bucharest, Bucharest 060042, Romania \\ ${ }^{2}$ Department of Physics, Technical "Gh. Asachi” University, 700050 Iasi, Romania \\ ${ }^{3}$ Department of Electronic Engineering, City University of Hong Kong, Hong Kong \\ ${ }^{4}$ Université de Lille, CNRS, UMR 8523, PhLAM-Physique des Lasers, Atomes et Molécules, CERLA-Centre d'Etudes et de Recherches \\ Lasers et Applications, F-59000 Lille, France
}

Correspondence should be addressed to Viorel-Puiu Paun; paun@physics.pub.ro

Received 6 August 2018; Accepted 6 August 2018; Published 1 November 2018

Copyright (c) 2018 Viorel-Puiu Paun et al. This is an open access article distributed under the Creative Commons Attribution License, which permits unrestricted use, distribution, and reproduction in any medium, provided the original work is properly cited.

This special issue of Complexity initially aimed at gathering leading-edge up-to-date studies showcasing the occurrence of fractal features in the dynamics of highly nonlinear complex systems. More than thirty years after coining term by Mandelbrot [1], fractals continue to fascinate the scientific community or the general public, with their wonderful propensity to infinitely repeat the same patterns at various (spatial and/or temporal) scales. On a more specialized ground, these continuous but nondifferentiable mathematical objects have become increasingly useful tools in developing original approaches to efficiently model natural or physical phenomena which escape from the traditional horizon of Euclidean metrics. Their range of applications is tremendously wide, from the well-known initial quest of measuring the length of Britain coast [2] to the most recent investigations on digital image processing for materials science [3], chaotic circuits [4], information technology [5], and medicine [6]. Initially developed at macro/mesoscopic scales, these objects penetrate now the nanoworld [7] with the development of ultrahigh resolution probes. At a temporal level, modeling of ultrafast phenomena (as peculiar oscillations evidenced in transient laser-generated plasmas [8]) can benefit from innovative scale relativity of fractal-based theoretical developments [9].

Although appealing, the initial fractal-oriented scope of this special issue has been proven to be quite sharp, and the decision was taken, in agreement with the journal editorial board, to broaden its range to a more general nonlinear dynamic field. In this context, 17 papers have been received for review, covering a wide variety of topics, from "pure" mathematics and theoretical physics to applications in materials science, plasma physics, medicine, and financial markets. Despite the high quality of all submitted papers, the very rigorous review process retained only seven of them for publications in this special issue. A brief presentation of each accepted paper is given in the following.

In "Analysis of Implicit Type Nonlinear Dynamical Problem of Impulsive Fractional Differential Equations," N. Ahmad et al. use a powerful combination of Caputo fractional derivatives and classical fixed point theorems (Banach and Krasnoselskii) to investigate the existence, uniqueness, and various kinds of Ulam-Hyers stability [10] of the solutions to impulsive fractional differential equations with nonlocal boundary conditions. A "pure" mathematical object, the Ulam concept of stability [10], finds quite significant applications in real-world problems like the formation of rogue waves (tsunami) in the oceans or the design of ultrahigh precision optical clocks. A very recent example is offered by the observation in optical fibers of the broken symmetry of Fermi-Pasta-Ulam recurrence [11]. In another "mathematical" paper of this special issue ("Moderate Deviations for Stochastic Fractional Heat Equation Driven by Fractional Noise"), X. Sun et al. use a fractional Laplacian operator to study a class of stochastic fractional heat equations driven by fractional noise. A central limit theorem is given, and a moderate deviation principle is established. 
Two papers in this special issue focus on plasma physics subjects. Plasmas are typical examples of strongly nonlinear dynamic systems, with many degrees of freedom, favorable for the development of (self-) ordered structures, instabilities, and (reversible) transitions from ordered to chaotic states. Complex space charge structures such as fireballs, multiple double layers, plasma bubbles, and solitons offer exciting study grounds for both experimentalists and theoreticians. C. Ursu et al. in "Fractal Method for Modeling the Peculiar Dynamics of Transient Carbon Plasma Generated by Excimer Laser Ablation in Vacuum" propose a fractal approach to simulate the formation of (surprising) V-shape radiating plasma structures (consisting of two lateral arms of high optical emissivity and a fast expanding central part of low emissivity), which have been previously observed [12]. In their model, the complexity of the interactions between the transient plasma particles (in the Euclidean space) is substituted by the nondifferentiability (fractality) of the motion curves to the same particles, but in a fractal space. For plane symmetry and particular boundary conditions, stationary geodesic equations at a fractal-scale resolution give a fractal velocity field with components expressed by means of nonlinear solutions (soliton-type, kink-type, etc.). The theoretical model successfully reproduces the formation of $\mathrm{V}$-shape radiating plasma structures experimentally observed. Another example on the usefulness of fractional-order modeling is offered by M. Guo et al. in "Study of Ion-Acoustic Solitary Waves in a Magnetized Plasma Using the Three-Dimensional TimeSpace Fractional Schamel-KdV Equation." Most of the quantum hydrodynamic models (such as the Korteweg-de Vries [13]) used to date to describe the propagation of these waves in the plasma are integer-order models while fractional calculus has been rarely considered, most probably because of its nonlocal character. In the present paper, the authors take advantage of the multiscale analysis to obtain a new model called the time-space fractional Schamel-KdV (TSFSchamel-KdV). Other advanced tools, such as Lie symmetry analysis, Riemann-Liouville fractional derivative, and multisoliton solutions derived through the Hirota bilinear method, are used to fully explore the characteristics of the ionacoustic solitary waves.

High current interest shape memory alloys (SMA) exhibit temperature- and stress-induced phase transformations which promote their use as both a sensor and an actuator, with prodigious practical applications in biomedical, robotics, automotive, and aerospace. Stress-strain hysteretic effects in these advanced functional materials lead to nonlinearity. Experimental dynamic analysis of SMAs was carried out and found that nonlinearity in phase transformation leads to a complicated dynamic behavior of the system. In this context, because of the nonlocal property (i.e., the next state of a system depends not only on its current state but also on all of its historical states), fractional calculus can provide better (or more realistic) results, for instance, in revealing the chaotic behavior (sometimes even for orders inferior to 3 ). Using a fractional-order approach, K. Rajagopal et al. reveal in the paper "Bifurcation and Chaos in Integer and Fractional Order Two-Degree-of-Freedom Shape Memory Alloy Oscillators" some periodic, quasiperiodic, chaotic, and hyperchaotic oscillations of a SMA-based oscillator for selected values of the excitation parameters and operating temperatures. The Grünwald-Letnikov derivative is used to derive the fractional-order model of the oscillator, and bifurcation plots are derived to study its dynamical behavior. Compared to the integer-order model, the fractionalorder model shows a more complex chaotic behavior, with also more extended regions of chaos.

In recent years, the fractal and multifractal analysis in biomedical data has seen a growing interest. Using a 2D technique of multifractal denoising, Y. Karaca et al. analyzed the data of 2204 individuals kept under observation at Massachusetts Medical School, University of Worcester, Massachusetts, USA, in their paper "Stroke Subtype Clustering by Multifractal Bayesian Denoising with Fuzzy $C$ Means and $K$-Means Algorithms." Such a high-dimension database may contain subtle details, not easily detectable by human observation. For the stroke subtype diagnosis, details are important including hidden information concerning the possible existence of medical history, laboratory results, and treatments. In the present paper, $2 \mathrm{D}$ multifractal denoising techniques and $K$-means and FCM algorithms applied to numeric data obtained from the attributes belonging to patients with seven different stroke subtypes. The newly proposed $2 \mathrm{D}$ multifractal Bayesian denoising technique (2D mBd) was revealed to be the most successful feature descriptor in each stroke subtype dataset, when compared to the existing literature.

The only paper of this special issue not making use of fractional calculus is "Information Feedback in Temporal Networks as a Predictor of Market Crashes" by S. Begušić et al. They use information theory (nonparametric measures) to study cross-sectional time-lagged dependencies and estimate directed temporal dependency networks in financial markets and real estate data. By analyzing the emergence of strongly connected feedback components in the estimated networks, the authors hypothesize that the existence of information feedback in financial networks induces strong spatiotemporal spillover effects and thus indicates a systemic risk. A systemic risk indicator is built and is shown to be useful in obtaining early warning signals for crashes in financial markets.

The variety of application fields and the richness of the submitted papers highlight, once more, the universality of the nonlinear dynamics conceptual approaches, at various levels, from nano- to galactic scale, from attoseconds to astronomical time units. From this perspective, complexity remains a challenging notion for theoretical modeling, technical analysis, and numerical simulation, in physics and mathematics (among others), because highly correlated nonlinear phenomena evolving over a wide range of timescales and length scales control the underlying systems and processes in their spatiotemporal evolution. The papers gathered here are just a few examples of this complexity, illustrating current efforts from different research groups for a better understanding of fascinating nonlinearity manifestations.

Last but not least, the guest editors would like to express their gratitude to the highly committed editorial office and to the voluntary reviewers, who ensured the success of this special issue. 


\title{
Conflicts of Interest
}

The editors declare that they have no conflicts of interest regarding the publication of this Special Issue.

\author{
Viorel-Puiu Paun \\ Maricel Agop \\ Guanrong Chen \\ Cristian Focsa
}

\section{References}

[1] B. Mandelbrot, Les Objets Fractals: Forme, Hasard et Dimension, Flammarion, Paris, 1975.

[2] B. Mandelbrot, "How long is the coast of Britain? Statistical self-similarity and fractional dimension," Science, vol. 156, no. 3775, pp. 636-638, 1967.

[3] V. Paun, "Fractal surface analysis of Zircaloy-4 SEM micrographs using the time-series method," Open Physics, vol. 7, no. 2, 2009.

[4] J. Lu and G. Chen, "Generating multiscroll chaotic attractors: theories, methods and applications," International Journal of Bifurcation and Chaos, vol. 16, no. 4, pp. 775-858, 2006.

[5] M. Agop, A. Gavriluț, V. Păun et al., "Fractal information by means of harmonic mappings and some physical implications," Entropy, vol. 18, no. 5, 2016.

[6] P. Postolache, Z. Borsos, V. A. Paun, and V. P. Paun, "New way in fractal analysis of pulmonary medical images," UPB Scientific Bulletin, Series A: Applied Mathematics and Physics, vol. 80, no. 1, pp. 313-322, 2018.

[7] A. Bescond, J. Yon, F. X. Ouf et al., "Automated determination of aggregate primary particle size distribution by TEM image analysis: application to soot," Aerosol Science and Technology, vol. 48, no. 8, pp. 831-841, 2014.

[8] C. Focsa, S. Gurlui, P. Nica, M. Agop, and M. Ziskind, "Plume splitting and oscillatory behavior in transient plasmas generated by high-fluence laser ablation in vacuum," Applied Surface Science, vol. 424, pp. 299-309, 2017.

[9] M. Agop, P. E. Nica, S. Gurlui, C. Focsa, V. P. Paun, and M. Colotin, "Implications of an extended fractal hydrodynamic model," The European Physical Journal D, vol. 56, no. 3, pp. 405-419, 2010.

[10] S. M. Ulam, A Collection of Mathematical Problems, Interscience, New York, NY, USA, 1960.

[11] A. Mussot, C. Naveau, M. Conforti et al., "Fibre multi-wave mixing combs reveal the broken symmetry of Fermi-PastaUlam recurrence," Nature Photonics, vol. 12, no. 5, pp. 303308, 2018.

[12] C. Ursu, P. Nica, and C. Focsa, "Excimer laser ablation of graphite: the enhancement of carbon dimer formation," Applied Surface Science, vol. 456, pp. 717-725, 2018.

[13] H. Washimi and T. Taniuti, "Propagation of ion-acoustic solitary waves of small amplitude," Physical Review Letters, vol. 17, no. 19, pp. 996-998, 1966. 


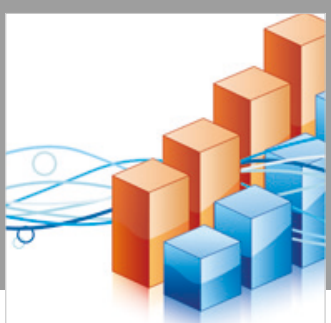

Advances in

Operations Research

\section{-n-m}
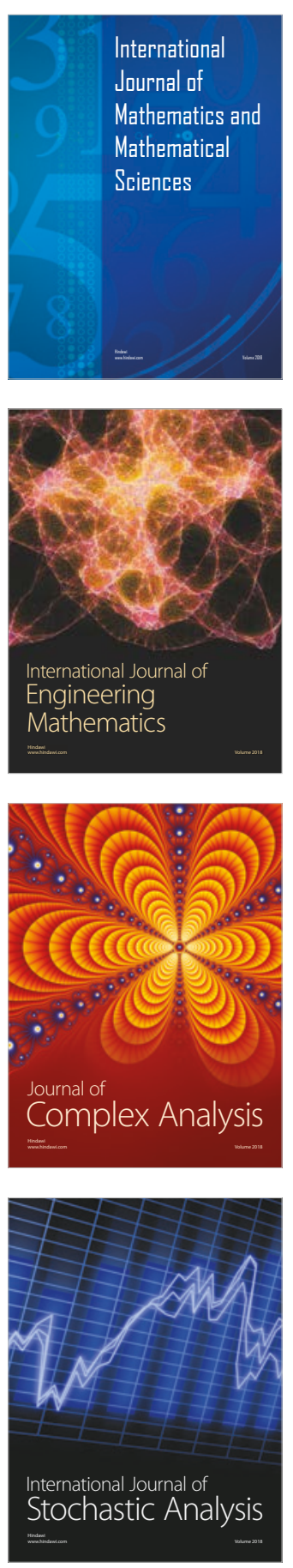
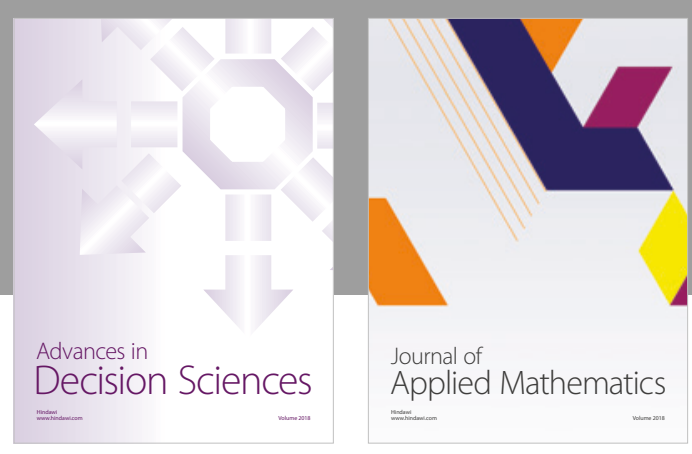

Journal of

Applied Mathematics
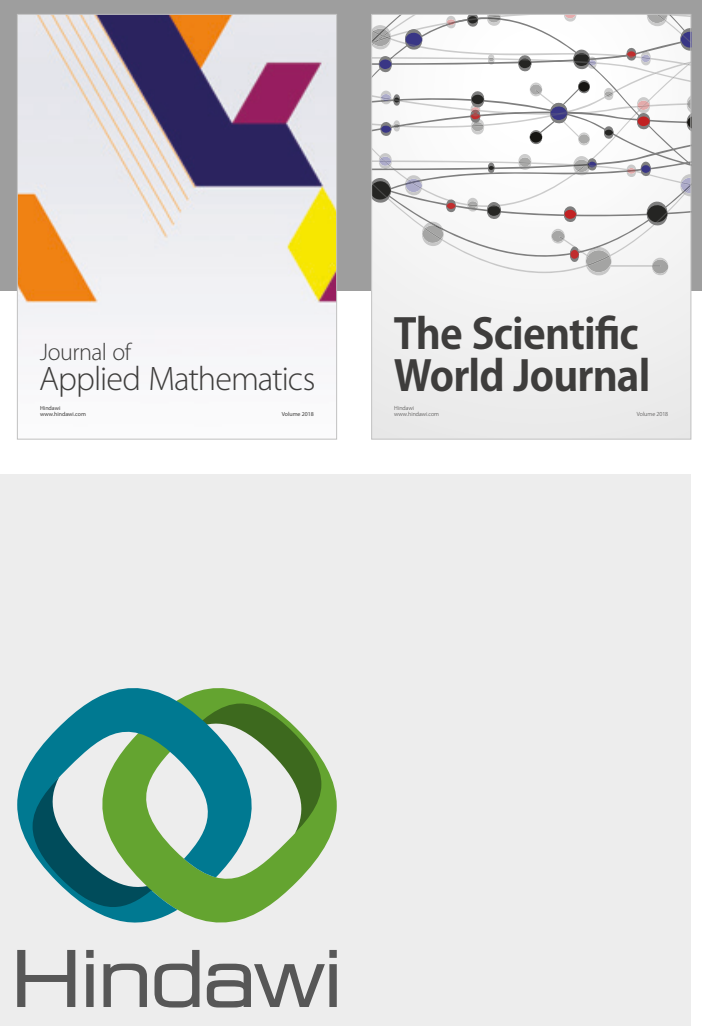

Submit your manuscripts at

www.hindawi.com

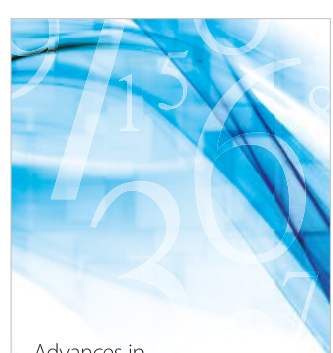

Advances in
Numerical Analysis
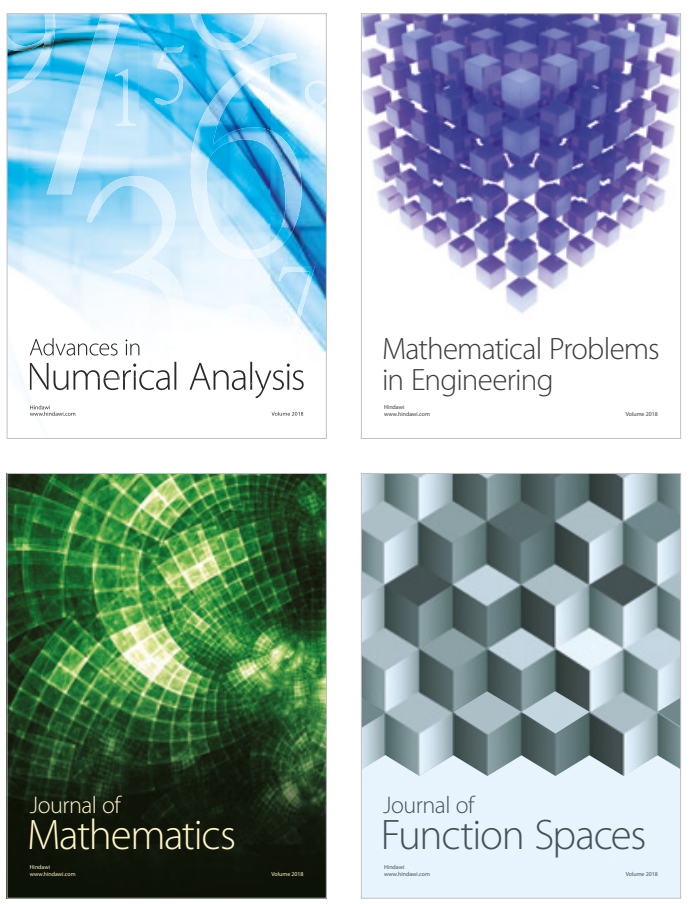

Mathematical Problems in Engineering

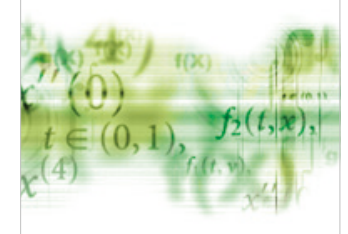

International Journal of

Differential Equations

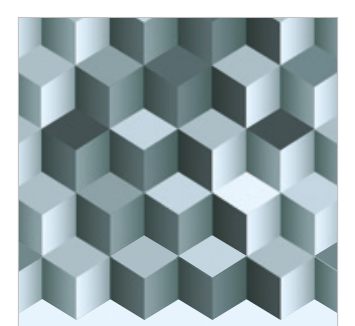

Journal of

Function Spaces
The Scientific

World Journal

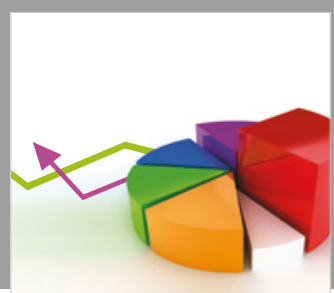

Journal of

Probability and Statistics
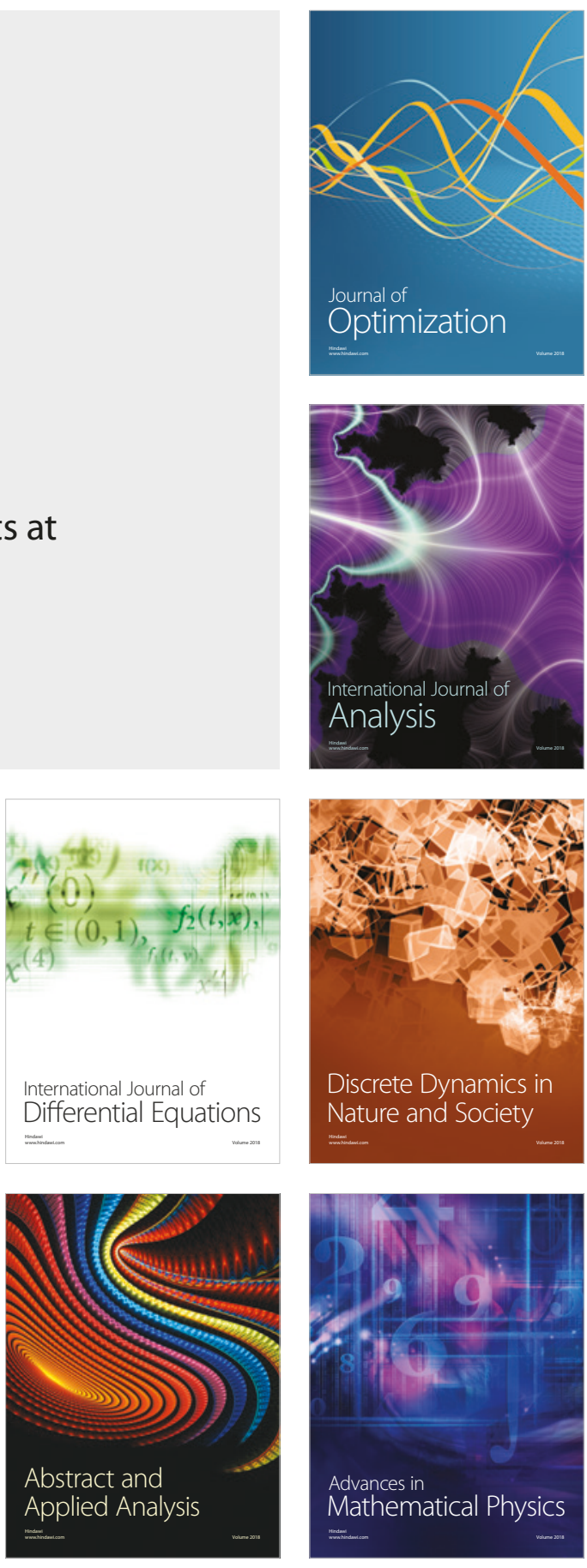